\title{
Memoria de trabajo, mecanismos inhibitorios y rendimiento lecto-comprensivo en grupos de comprendedores de secundaria básica
}

\author{
Working Memory, inhibitory processes and reading comprehension performance in groups of \\ comprehenders of secondary school
}

\author{
María Silvina Demagistri ${ }^{1}{ }^{*}$, Lorena Canet ${ }^{1}$, Liliana Naveira ${ }^{1}$, María Richard's ${ }^{1}$
}

1 Facultad de Psicología. Universidad Nacional de Mar del Plata. Mar del Plata, Argentina.

\section{Resumen}

La comprensión lectora es un proceso complejo que supone la intervención de un conjunto de variables interrelacionadas. Diversos estudios han planteado el rol de la memoria de trabajo y los mecanismos inhibitorios en la habilidad de comprender un texto. El propósito del presente trabajo es doble: por un lado, se propone evaluar las relaciones entre memoria de trabajo, mecanismos inhibitorios y comprensión lectora en adolescentes de primer año de la Educación Secundaria Básica (E.S.B.) de 12 y 13 años de edad, distribuidos en dos grupos de desempeño en comprensión lectora; y por otro, analizar las diferencias en los rendimientos ejecutivos de memoria de trabajo y mecanismos inhibitorios en función del nivel de comprensión lectora (alto y bajo rendimiento). Para ello, se administraron un conjunto de tareas para evaluar: comprensión de textos, fluidez lectora, habilidades verbales, memoria de trabajo y mecanismos inhibitorios. Los resultados señalan correlaciones entre rendimiento lecto-comprensivo, memoria de trabajo, mecanismos inhibitorios (supresión y restricción) y habilidades verbales. A su vez, la evidencia señala que los grupos de desempeño en comprensión lectora presentan diferencias estadísticamente significativas en sus correspondientes rendimientos en memoria de trabajo e inhibición.

Palabras clave: comprensión lectora, memoria de trabajo, mecanismos inhibitorios, adolescentes

\begin{abstract}
The process of reading comprehension involves a group of interrelated variables. Several studies have presented the role of working memory and the inhibition process in reading comprehension skills. The aim of this research is double, on one side to assess the relationship between working memory, inhibitory processes and reading comprehension in adolescents belonging to the first year of secondary school of 12 and 13 years old, in two groups of comprehension levels; and on the other hand, to analyze the differences in executive performance of working memory and inhibitory processes in different levels of reading comprehension (high and poor performance). A number of tests were carried out to assess the level of reading comprehension, verbal skills, reading fluency, working memory and inhibitory processes. Results show that levels of reading comprehension, working memory, inhibitory processes (deletion and restraint) and verbal skills are related. The evidence suggests that the groups of different levels of reading comprehension show significant differences in their working memory, inhibitory processes and verbal skills performance.
\end{abstract}

Keywords: reading comprehension, working memory, inhibitory processes, adolescents

\footnotetext{
* Correspondencia: msdemagistri@gmail.com. Facultad de Psicología, Universidad Nacional de Mar del Plata, Funes 3250, Cuerpo V- Nivel III. Teléfono: +54 (0223) 4752526

Recibido: 16-05-12. Revisión desde: 16-05-12. Aceptado: 28-06-12
}

DOI: $10.5839 / \mathrm{rcnp} .2012 .0702 .06$ 


\section{Introducción}

La comprensión lectora $(\mathrm{CL})$ representa uno de los problemas fundamentales en el estudio de la cognición humana (Kintsch, 1988). La misma permite la adquisición de información sobre el mundo real y ficcional, posibilitando además la comunicación y el éxito académico (Cain \& Oakhill, 2007).

La CL ha sido descripta como una variable compleja mediatizada por diversas habilidades y procesos cognitivos. Su realización abarca procesamientos lingüísticos propios de los niveles del lenguaje: léxico, morfo-sintáctico, semántico y pragmático. A su vez, implica que el lector-comprendedor ponga en marcha procesos cognitivos y metacognitivos de diverso orden (Cain, Oakhill \& Bryant, 2004; Gernsbacher, 1990; Kintsch, 1988, 1998).

Desde que Baddeley \& Hitch (1974) y Baddeley (1986) propusieron el concepto de memoria de trabajo (MT), conceptualizada como un sistema activo de almacenamiento temporario y procesamiento simultáneo y activo de la información, este constructo teórico se ha vuelto necesario en la explicación de los procesos cognitivos superiores como el aprendizaje, el razonamiento o la comprensión. En particular, se sugiere que la comprensión de un texto requiere una MT donde depositar los productos resultantes del procesamiento de las distintas frases (proposiciones textuales e inferidas) al mismo tiempo que se realizan los procesos exigidos por la lectura y la tarea en curso.

Desde el modelo de Baddeley (1986) la MT es considerada como un sistema complejo, constituido por subsistemas que cumplen una multiplicidad de funciones. Se le atribuyen tres componentes: el ejecutivo central, encargado de los recursos del procesamiento de dos subsistemas subsidiarios; un bucle articulatorio, encargado del almacenamiento de la información verbal; y una agenda o registro viso-espacial, encargada del almacenamiento visual y espacial. Baddeley \& Wilson (2000) sumaron a este modelo un buffer episódico, de capacidad limitada, dedicado a integrar información de diversas fuentes y construir modelos mentales.

Siguiendo estos postulados, diversas investigaciones estudiaron la relación entre MT y $\mathrm{CL}$ (Cutting, Materek, Cole, Levine \& Mahone, 2009; Daneman \& Carpenter, 1980; De Beni, Borella \& Carretti, 2007; De Beni, Pazzaglia, Gyselinck \& Meneghetti, 2005; Gutiérrez, García Madruga, Elosúa, Luque \& Garate, 2002; Swanson \& O'Conner, 2009). Sin embargo, los estudios existentes estudiaron la población infantil y adulta, siendo menos los reportes sobre población adolescente.

En el estudio de dicha relación, un hito importante lo constituyen los trabajos de Daneman \& Carpenter (1980). Las autoras introdujeron la prueba de amplitud de MT como una medida de su funcionalidad durante la lectura. La modalidad de la prueba puede ser visual, Reading Span Test, o auditiva, Listening Span Test (LST). La prueba consiste en leer o escuchar oraciones, atribuir verdad o falsedad al contenido de las mismas $y$, luego, nombrar la última palabra de cada una en el orden en que fueron leídas o escuchadas. Los estímulos van aumentando en cantidad (una oración, dos, tres y así hasta llegar a seis). EI número de palabras que cada sujeto puede recordar y evocar se toma como medida de amplitud de MT (span). Esta prueba reproduce las exigencias de la lectura. En principio, requiere un procesamiento de la información, es decir: el sujeto debe construir una representación mental del estímulo; luego, implica que cierta información sea sostenida en la memoria y finalmen- te, que ambas se coordinen para emitir la respuesta requerida (Abusamra, Cartoceti, Raiter, \& Ferreres, 2008).

En esta línea se considera a la comprensión como un proceso de integración activa de información, por lo cual las investigaciones actuales se encaminan a evaluar el papel de la MT y de los procesos de inhibición en este proceso cognitivo. Para cumplir con el objetivo de crear una representación mental coherente del texto, el lector debe sostener cierta información en la MT mientras computa las relaciones entre palabras y oraciones, a su vez, debe poder inhibir la información irrelevante y actualizar la información relevante en su MT (Abusamra, et al., 2008).

Se sabe además que la MT presenta variabilidad individual y es clave en el desarrollo cognitivo. El rendimiento en MT sufre un incremento en su funcionalidad a través de la edad y presenta una declinación gradual a través de la adultez hacia los 50 años (Chiappe, Hasher \& Siegel, 2000). Chiappe et al. (2000) señalan que la MT puede ser considerada como un conjunto de recursos atencionales limitados o un espacio finito de trabajo que debe compartirse entre el procesamiento y el almacenamiento. Por tanto, si las demandas de una tarea aumentan, en el ejecutivo central quedan menos recursos disponibles para los sistemas subsidiarios.

En esta línea, Chiappe et al. (2000) retoma investigaciones de Hasher y colegas (Hasher \& Zacks, 1988; Hasher, Lustig, \& Zacks, 2007; Hasher, Tonev, Lustig \& Zacks, 2001; Zacks \& Hasher, 1994), quienes suman un aspecto clave en el estudio de las relaciones entre MT y habilidades lectoras. Estas autoras hipotetizan que los déficits cognitivos relativos a la edad y posiblemente las diferencias individuales asociadas a las habilidades lectoras pueden deberse al ineficiente control inhibitorio de la atención. Postulan un modelo que plantea la existencia de tres procesos inhibitorios: acceso, borrado y restricción. La función de acceso es la encargada de controlar el ingreso de la información irrelevante al foco atencional. Cuando esta función falla la información irrelevante entra al foco dificultando el procesamiento de la información relevante. El borrado se ocupa de suprimir la información irrelevante de la conciencia, que puede haber eludido el control de la función de acceso y sólo posteriormente, haber sido reconocida como irrelevante o también puede haber sido relevante en una situación previa pero como consecuencia del cambio en los objetivos ya no lo es. Por último, la restricción consiste en la supresión de respuestas preponderantes pero inapropiadas. Este tipo de inhibición puede suprimir errores de interpretación de textos y del lenguaje (Chiappe, et al., 2000).

En el campo de las dificultades de lectura, el estudio de Chiappe et al. (2000) señalan que los déficits en la MT y en los mecanismos de inhibición son característicos de los individuos con dificultades en la lectura en todas las edades. A su vez, suponen que los déficits en la supresión de información pueden estar asociados a los niveles de span. En esta línea, Hasher et al. (2007) enfatizan que el estudio de la MT debe focalizarse en los procesos ejecutivos más que en los procesos de almacenamiento y procesamiento. Afirman que la variabilidad de la MT tiene su fuente en el rol de los procesos inhibitorios en el ejecutivo central. Enfatizan que estos procesos permiten mantener la conciencia libre de información irrelevante, que puede llegar a impedir la resolución eficiente de tareas. Sostienen que las diferencias en el rendimiento de span o en lectura se deberían a la habilidad de restringir la atención hacia lo que es relevante 
más que a la habilidad de activar conceptos relevantes. En este marco, diversos trabajos de investigación se han focalizado en las diferencias entre comprendedores de alto y bajo nivel en cuanto a su capacidad de inhibir información irrelevante y actualizar la información relevante (Abusamra, et al., 2008; Borella, Carretti, Pelegrina, 2010; Canet-Juric, Urquijo, Richard's \& Burín, 2009; Carretti, Borella, Cornoldi, \& De Beni, 2009; Chiappe, et al. 2000).

En este contexto, el presente trabajo plantea evaluar las relaciones entre $\mathrm{MT}$, mecanismos inhibitorios y $\mathrm{CL}$ en adolescentes de primer año de la E.S.B. En segundo término, se propone detectar niveles de desempeño en $\mathrm{CL}$ y analizar si los grupos presentan diferencias estadísticamente significativas en sus correspondientes rendimientos en MT y mecanismos inhibitorios.

La presente investigación supone que existen correlaciones fuertes y estadísticamente significativas entre $\mathrm{CL}, \mathrm{MT}$ y mecanismos inhibitorios. Sostiene igualmente que existen diferencias estadísticamente significativas entre los grupos de desempeño de alto y bajo nivel en $\mathrm{CL}$ y sus correspondientes rendimientos en $\mathrm{MT}$ e inhibición, considerada ésta última según las funciones de acceso, borrado y restricción. Así, los sujetos que obtienen puntuaciones altas en $\mathrm{CL}$ serían aquellos que logran buenos desempeños en las pruebas de MT e inhibición.

\section{Método}

\section{Diseño y Participantes}

El tipo de estudio utilizado fue correlacional, con un diseño no experimental transversal, e hipótesis de diferencia de grupos.

Se evaluaron 53 alumnos de dos grupos conformados de primer año de la E.S.B., de una escuela pre- universitaria de la ciudad de Mar del Plata. (Ver características descriptivas de la muestra en Tabla 1).

De acuerdo al rendimiento lecto-comprensivo se seleccionaron 20 sujetos distribuidos en dos grupos en función del siguiente criterio: 1) El grupo de comprendedores con rendimiento alto $(n=12)$, conformado por sujetos que mostraron un rendimiento, al menos, un desvío estándar por sobre la media grupal; 2) El grupo de comprendedores con rendimiento bajo $(n=8)$, constituido por sujetos cuyo desempeño se ubicó al menos un desvío estándar por debajo de la media grupal. A su vez, ambos grupos presentaron el mismo nivel en sus habilidades de decodificación lectora $(t(18)=2,06 ; p>.05)$.

\section{Instrumentos}

Los objetivos del estudio implicaron la selección de un conjunto de tareas. Se administraron las siguientes pruebas:

- Prueba de CL diseñada para el nivel educativo. Consiste en la lectura silente de un texto y la resolución de diez preguntas abiertas. (Ávalos \& Díaz, 1997).

- Fluidez lectora: Sub-test de la E.N.I., Velocidad de lectura de un texto en voz alta (Matute, Rosselli, Ardila, \& OstroskySolís, 2007). Se presenta al sujeto un texto (Tontolobo y el carnero) que debe leer en voz alta. Se computa el tiempo total de lectura.

- Habilidades Verbales: Sub-test de vocabulario del WISC III (Weshler, 1991). La tarea consiste en solicitar al sujeto que recupere el significado de un conjunto de palabras.
Tabla 1. Características de la muestra $(n=53)$ y sub-muestra $(n=20)$ del estudio.

\begin{tabular}{|c|c|c|c|}
\hline & $n=53$ & CRA* $^{*}(n=12)$ & $\mathrm{CRB}^{* *}(\mathrm{n}=8)$ \\
\hline \multicolumn{4}{|l|}{ Sexo } \\
\hline Femenino & $60 \%$ & $83 \%$ & $75 \%$ \\
\hline Masculino & $40 \%$ & $17 \%$ & $25 \%$ \\
\hline \multicolumn{4}{|l|}{ Edad } \\
\hline M & 12.2 & 12.08 & 12.07 \\
\hline $\mathrm{DE}$ & .277 & .017 & .035 \\
\hline Edad mínima & 12.1 & 12.06 & 12.02 \\
\hline Edad máxima & 13.1 & 12.11 & 12.10 \\
\hline
\end{tabular}

Nota. ${ }^{*}$ Comprendedores de rendimiento alto. ${ }^{* *}$ Comprendedores de rendimiento bajo

Para evaluar rendimientos ejecutivos se aplicaron las tareas de:

- MT: Tarea de amplitud de palabras: perteneciente a la Batería de Evaluación de MT (AWMA) adaptada al español (Injoque-Ricle, Calero, Alloway \& Burín, 2011). La prueba aporta dos puntajes: span y puntaje total. Este último se obtiene por la sumatoria de todos los aciertos a lo largo de la prueba. Adicionalmente, se tomaron los errores de intrusión como medidas indirectas de inhibición. Los errores se clasifican siguiendo a Chiappe et al. (2000): - palabras del ensayo en curso pero no target (NT), que muestran una deficiencia en la inhibición como resultado de un aumento en la entrada de información irrelevante; - palabras de listas anteriores (LP), que reflejan interferencia proactiva que puede ser el resultado de fallos en la inhibición de borrado de información irrelevante; - palabras extrañas que no aparecían en ningún ensayo (EX), que reflejan deficiencias en la función de restricción de la inhibición.

- Inhibición: Test de Hayling (adaptación de Cartoceti, Sampedro, Abusamra, \& Ferreres, 2009). Evalúa los procesos de iniciación y supresión, utilizando estímulos verbales. El instrumento consta de dos secciones diferentes. En la primera, el sujeto debe completar 15 oraciones en que falta la última palabra, con un estímulo que sea consistente con el contexto sintáctico-semántico (respuesta de iniciación). En la segunda, se requiere el completamiento de las oraciones con una palabra inconsistente con el contexto oracional (respuesta de supresión). Para la variable Inhibición se considera: tiempo de latencia en la prueba de iniciación (Iniciación); tiempo de latencia en la prueba de supresión (Supresión); puntaje de inhibición, este ítem asigna un puntaje que pena la proximidad semántica de la respuesta con la oración propuesta es decir, evalúa la dificultad de inhibir la respuesta dominante (Restricción).

\section{Procedimiento}

En una primera etapa, se acordó con la escuela la realización del trabajo y se solicitó el consentimiento informado a los padres o cuidadores de los alumnos y el asentimiento de los participantes. Se explicó detalladamente en qué consistía la investigación garantizándose la confidencialidad de la información obtenida y su utilización con fines exclusivamente científicos bajo la Ley Nacional 25.326 de protección de los datos personales. Durante la segunda etapa, se administró colectivamente el instrumento para evaluar CL. A su vez, se tomaron en sesiones individuales, las pruebas de vocabulario, fluidez lectora, MT e inhibición. 
Demagistri, M. S., Canet, L., Naveira, L., Richard's, M. Rev. chil. neuropsicol. 7(2): 72-78, 2012

Tabla 2. Correlación de Pearson entre pruebas administradas $(n=20)$.

\begin{tabular}{|c|c|c|c|c|c|c|c|c|c|}
\hline & 2 & 3 & 4 & 5 & 6 & 7 & 8 & 9 & 10 \\
\hline 1.Prueba de comprensión & $.647(* *)$ & $.490\left(^{*}\right)$ & $.581\left(^{* *}\right)$ & NS & NS & NS & NS & $-.529 \quad(*)$ & $-.582(* *)$ \\
\hline 2. Habilidades verbales & - & $.675\left(^{* *}\right)$ & $.711\left(^{* *}\right)$ & NS & NS & NS & NS & NS & $-.548\left(^{*}\right)$ \\
\hline \multicolumn{10}{|l|}{ Memoria de trabajo } \\
\hline 3.Span & & - & $.858(* *)$ & NS & NS & NS & $-.512(*)$ & NS & NS \\
\hline 4.Puntaje total & & & - & NS & NS & NS & NS & NS & NS \\
\hline \multicolumn{10}{|l|}{ Errores de intrusión } \\
\hline 5.NT & & & & - & NS & NS & $.500\left(^{*}\right)$ & NS & NS \\
\hline 6.LP & & & & & - & NS & NS & NS & NS \\
\hline 7.EX & & & & & & - & NS & NS & NS \\
\hline \multicolumn{10}{|l|}{ Inhibición } \\
\hline 8.Iniciación & & & & & & & - & NS & NS \\
\hline 9.Supresión & & & & & & & & - & $.473\left({ }^{*}\right)$ \\
\hline 10.Restricción & & & & & & & & & - \\
\hline & & & & & \multirow{2}{*}{\multicolumn{3}{|c|}{$\begin{array}{c}\text { CRA } \\
n=12\end{array}$}} & \multirow{2}{*}{\multicolumn{2}{|c|}{$\begin{array}{l}\text { CRB } \\
n=8\end{array}$}} \\
\hline & & & & & & & & & \\
\hline & & & & & $\mathrm{M}$ & & $\mathrm{DE}$ & $\mathrm{M}$ & $\mathrm{DE}$ \\
\hline Prueba de comprensión (Rt & tas/30) & & & & 23.53 & & 1.11 & 12.75 & 2.05 \\
\hline Habilidades verbales & & & & & 41,33 & & 4.07 & 34.75 & 3.54 \\
\hline \multicolumn{10}{|l|}{ MT } \\
\hline Span & & & & & 2.67 & & 0.49 & 2.13 & 0.35 \\
\hline Puntaje total & & & & & 16.41 & & 1.60 & 14 & 2.13 \\
\hline \multicolumn{10}{|l|}{ Errores de intrusión } \\
\hline NT & & & & & 0.08 & & 0.28 & 0.13 & 0.35 \\
\hline $\mathrm{LP}$ & & & & & 0.58 & & 0.66 & 0.25 & 0.46 \\
\hline EX & & & & & 0.33 & & 0.65 & 0.25 & 0.46 \\
\hline \multicolumn{10}{|l|}{ Inhibición } \\
\hline Iniciación & & & & & 1.24 & & 0.58 & 1.69 & 0.58 \\
\hline Supresión & & & & & 1.93 & & 1.33 & 3.19 & 1.83 \\
\hline Puntaje parte B & & & & & 2.50 & & 2.81 & 5.50 & 2.20 \\
\hline
\end{tabular}

Durante la tercera etapa, se detectó a grupos de comprendedores según su nivel de rendimiento en $\mathrm{CL}$ fuera alto o bajo y se procedió al análisis de los resultados.

\section{Resultados}

Para evaluar relaciones entre $\mathrm{MT}$, mecanismos inhibitorios y $\mathrm{CL}$ en adolescentes de primer año de la E.S.B. se aplicó el Coeficiente de correlación de Pearson. La Tabla 2 presenta los resultados $(n=20)$. Los datos permiten afirmar que el rendimiento en comprensión muestra relaciones estadísticamente significativas con MT (Span $=p<.05$ y Puntaje Total $=p<.01)$, inhibición de supresión $(p<.05)$, inhibición de restricción $(p<.01)$ y habilidades verbales $(p<.01)$. En cambio, los errores de intrusión no presentan dicha correlación.
Como se ha mencionado, los desempeños en CL permitieron conformar dos grupos según los niveles de rendimiento (+1 $D E /-1 D E$ ), equiparados en fluidez lectora. La Tabla 3 presenta el rendimiento de los grupos en cada una de las pruebas considerando $M$ y $D E$.

Para establecer la existencia de diferencias estadísticamente significativas entre los desempeños de cada grupo, se aplicó una prueba $t$ de Student. Posteriormente, con el objeto de evaluar la magnitud de las diferencias encontradas se utilizaron dos indicadores del tamaño del efecto: $d$ de Cohen y $r$ (Morales-Vallejo, 2009) calculados ambos a través del programa Effect Syze Calculator (Watkins, 2003). La Tabla 4 presenta los resultados indicando los niveles de significación estadística encontrados. 
Tabla 4. Prueba $t$ y tamaños de efecto $\left(E t a^{2}\right)$ sobre rendimiento en comprensión lectora, en función de grupos de alto y bajo nivel.

\begin{tabular}{|c|c|c|c|c|c|}
\hline & \multirow[t]{2}{*}{$t$} & \multirow[t]{2}{*}{ gl. } & \multirow{2}{*}{$\begin{array}{l}\text { Significación } \\
\text { (bilateral) }\end{array}$} & \multicolumn{2}{|c|}{$E t a^{2}$} \\
\hline & & & & $\begin{array}{c}d \\
\text { Cohen }\end{array}$ & $r$ \\
\hline Habilidades verbales & $\begin{array}{c}- \\
3.75\end{array}$ & 18 & $.001 * * *$ & -1.69 & .66 \\
\hline \multicolumn{6}{|l|}{ MT } \\
\hline Span & 2.67 & 18 & $.015^{* *}$ & -1.22 & .53 \\
\hline Puntaje total & $\begin{array}{c}- \\
2.89\end{array}$ & 18 & $.010 * *$ & -1.32 & .56 \\
\hline \multicolumn{6}{|l|}{$\begin{array}{l}\text { Errores de } \\
\text { intrusión }\end{array}$} \\
\hline NT & .28 & 18 & NS & .16 & .06 \\
\hline LP & $\begin{array}{c}- \\
1.22\end{array}$ & 18 & NS & -.55 & .27 \\
\hline EX & -.31 & 18 & NS & -.13 & .07 \\
\hline \multicolumn{6}{|l|}{ Inhibición } \\
\hline Iniciación & 1.68 & 18 & NS & .77 & .36 \\
\hline Supresión & 1.77 & 18 & NS & .81 & .38 \\
\hline Restricción & 2.53 & 18 & $.021^{*}$ & 1.15 & .51 \\
\hline
\end{tabular}

Nota. ${ }^{*} \mathrm{p}<.05 ;{ }^{* *} \mathrm{p}<.01 ;{ }^{* * *} \mathrm{p}<.001$. NS: No significativo.

Se hallaron diferencias estadísticamente significativas en las pruebas de habilidades verbales $(p<.001)$, MT (span $=p<$ .015 , puntaje total $=p<.010)$ e inhibición de restricción $(p<$ .021).

A su vez, no se hallaron diferencias significativas en errores de intrusión, iniciación y supresión. A pesar de ello, con respecto a las latencias, puede señalarse que ambos grupos incrementaron sus tiempos de respuestas en la prueba de supresión en comparación con la prueba de iniciación. El incremento del tiempo y la dificultad, mostraría el mecanismo específico encargado de evitar una respuesta automática y permitir una más alejada sintáctica y semánticamente. La media de los comprendedores de bajo nivel mostró un tiempo 1.20" más alto que la de los comprendedores de alto nivel, esto permitiría suponer un funcionamiento menos eficiente del mecanismo de supresión en este grupo.

\section{Discusión}

La presente investigación giró en torno a dos propósitos. En principio, buscó evaluar las relaciones entre rendimientos ejecutivos y diversos niveles de lecto-comprensión en adolescentes de primer año de la E.S.B. Los resultados dieron cuenta de un conjunto de correlaciones que permitirían describir la habilidad de comprender textos y caracterizar el funcionamiento cognitivo del grupo etario.

\section{Relaciones entre memoria de trabajo, mecanismos inhibitorios y comprensión lectora}

Primero, la relación $\mathrm{CL} /$ habilidades verbales, señalaría que acceder a un vocabulario amplio contribuiría a la construcción de representaciones coherentes del texto. A su vez, conocer el significado de las palabras y tener un acceso eficiente a las mismas implicaría poder destinar recursos cognitivos a otros procesos como la integración inferencial o el monitoreo, sin tener que "detenerse a pensar" qué significa cada palabra que va apareciendo en el texto. Estas afirmaciones se hallan en coincidencia con reportes previos que indican que el vocabulario es uno de los mejores predictores de la comprensión (CanetJuric, et al., 2009; Perfetti, 1985).

Segundo, la correlación CL/ MT permitiría sostener la hipótesis de que los procesos lectores, implican la realización simultánea de tareas. Es decir, es necesario procesar y almacenar eficientemente la información en función de la construcción mental del significado de aquello que se lee, tal como afirmaron investigaciones previas (Abusamra, et al., 2008; Cain \& Oakhill, 2006; Carretti, et al., 2009; Daneman \& Merikle, 1996; Gutierrez, et al., 2002).

Tercero, las relaciones inversas entre: CL/ inhibición de supresión; $\mathrm{CL} /$ inhibición por restricción, señalarían que, a mayor rendimiento en comprensión, menor es el tiempo requerido para suprimir información irrelevante y menor es la dificultad de restringir las respuestas prepotentes. De este modo, la evidencia indicaría que la capacidad de inhibir información irrelevante y activar sólo la relevante está implicada en el proceso comprensivo. Estos hallazgos son coincidentes con la literatura (Abusamra, et al., 2008; Canet-Juric, et al. (2009), Chiappe, et al., 2000; Daneman \& Carpenter, 1980; De Beni, et al., 2007).

En cuarto lugar, la correlación habilidades verbales/MT se explicaría en función de la prueba utilizada (LST), ya que esta mide la capacidad de procesamiento cognitivo mediante el procesamiento semántico/lingüístico. En esta tarea experimental es necesario contar con los significados requeridos, poder acceder a ellos a través de la MT, procesarlos y almacenar sólo los necesarios. Por lo tanto, el procesamiento de significados queda implicado en el rendimiento en MT. En relación a estas afirmaciones, Miyake \& Shah (1999) en una síntesis sobre diversos modelos de MT, señalaron una línea teórica que postula el rol central del conocimiento a largo plazo (en este caso semántico) en el rendimiento en MT. En este sentido, la evidencia hallada realizaría aportes a favor de esta relación.

En quinto lugar, se observó la relación inversa habilidades verbales/inhibición por restricción. Cabe aclarar que la tarea de restricción considera la capacidad de emitir una respuesta alejada al contexto sintáctico/semántico del estímulo. Por ello, al aumentar los recursos lingüísticos menor es la dificultad de emitir respuestas alejadas al contexto. Ello permitiría plantear la hipótesis de que en las tareas verbales como la lectura, el control inhibitorio se realizaría de un modo más eficiente según aumenten los recursos lingüísticos.

En sexto lugar, la relación Iniciación /NT, es decir, errores de acceso, señalaría que ambas pruebas evalúan de alguna manera la entrada de la información al foco atencional de la MT (Hasher et al., 2007 p. 231). A su vez, la relación inversa Iniciación/MT, indicaría que a menor tiempo de reacción en una tarea cognitiva mayor es la amplitud de la MT. Esto daría cuenta de la importancia de la velocidad de procesamiento en el inicio de tareas cognitivas, en el procesamiento de estímulos y, por lo tanto, en la liberación de recursos atencionales.

Por último, la correlación Supresión/ Restricción, estaría indicando que las funciones inhibitorias encargadas de dirigir la atención a lo relevante e impedir que conceptos irrelevantes interfieran en los procesamientos se encuentran vinculadas. 
Diferencias en los rendimientos ejecutivos de memoria de trabajo y mecanismos inhibitorios en función del nivel de comprensión lectora

Con respecto al segundo propósito del estudio, referido a establecer diferencias entre distintos grupos de comprendedores en relación a indicadores ejecutivos, se procedió como primera medida a equiparar a los grupos en su habilidad de decodificación. Por ello, en este estudio los sujetos se agruparon verificando que se diferenciaran en sus rendimientos en comprensión lectora y no en su capacidad de decodificación. El análisis de los resultados permitió mostrar estas diferencias: - los comprendedores con un alto nivel de rendimiento se caracterizan por contar con mejores habilidades verbales, poseer una mayor amplitud de MT y lograr detener más eficientemente las respuestas dominantes pero inadecuadas; - los comprendedores de bajo nivel de rendimiento, en cambio, se caracterizan por desempeños más pobres en vocabulario, menor eficiencia en los procesos de almacenamiento y mantenimiento de la información en el foco atencional y un rendimiento más bajo en el mecanismo inhibitorio de restricción. Esto se halla en coincidencia con lo afirmado por Hasher et al. (2007) cuando explica que los grupos de sujetos con diferencias en MT o habilidades lectoras difieren fundamentalmente en la habilidad para mantener activa la información relevante y restringir la atención a ella y no en la activación de la información.

Si bien los resultados confirmaron diferencias significativas entre los grupos de rendimiento estos datos no son suficientes para contrastar la hipótesis nula de no diferencia entre los grupos y tampoco permiten comparar directamente entre sí las diferencias encontradas. Tal conclusión sólo puede realizarse si se toman en cuenta los tamaños del efecto. Siguiendo las orientaciones de Cohen (en Morales-Vallejo, 2009) los tamaños del efecto indicaron magnitudes altas para las variables: habilidades verbales, MT e inhibición por restricción. En cambio, presentaron magnitudes de efecto moderadas las variables de LP (errores de borrado), iniciación y supresión. Las variables NT (acceso) y EX (restricción) presentaron tamaños de efectos leves. Estos resultados permiten afirmar que: -a pesar de la ausencia de diferencias estadísticamente significativas en errores de intrusión de borrado (LP) y supresión, la magnitud moderada del tamaño del efecto hablaría de la incidencia de este mecanismo sobre el rendimiento en lecto-comprensión; - en cambio, las diferencias en las medidas de restricción podrían explicarse considerando las características de las pruebas administradas, el Test de Hayling mide esta función en forma directa en cambio los errores de intrusión (EX) son medidas indirectas de dicha función inhibitoria.

Lo señalado hasta aquí se halla en consonancia con la afirmación de que los déficits en MT e inhibición son característicos de los individuos con dificultades de lectura en todas las edades (Chiappe, et al., 2000). A su vez, permite seguir explorando la hipótesis de que la inhibición no es una función unitaria sino que se compone de procesos disociables que son parte del funcionamiento de la MT. Esto debido a que las distintas tareas utilizadas en la evaluación de los mecanismos inhibitorios muestran efectos diferentes sobre la comprensión, pudiendo discriminar entre la función de acceso, supresión y restricción.

Por último, los hallazgos señalan la complejidad de la $\mathrm{CL}$ y ponen de manifiesto la incidencia de un conjunto de funciones y procesos. Aportan evidencia en torno a las diferencias en ren- dimientos ejecutivos en grupos de comprendedores de E.S.B. y presentan los efectos diferenciales que ellos ejercen.

\section{Referencias}

Abusamra, V., Cartoceti, R., Raiter A., \& Ferreres A. (2008). Una perspectiva cognitiva en el estudio de la comprensión de textos. Psico Porto Alegre, PUCRS, 39(3), 352-361.

Abusamra, V., Ferreres, A., Raiter, A., De Beni R., \& Cornoldi, C. (2010). Test Leer para comprender TCL. Evaluación de la comprensión de textos. Bs. As.: Paidós.

Álvarez Hernández, A., Catuogno, M., Inchausti, B., \& Naviera, L. (2002). Aprender a comprender. De los procesos de semiosis textual a la lectura como función social. Argentina: Martín.

Ávalos, M. \& Díaz, A. (1997). Hacia una evaluación personalizada de la comprensión lectora. Lingüistica en el aula, 1(1), pp.11-43. Universidad Nacional de Córdoba. Centro de Investigaciones Lingüísticas.

Baddeley, A. (1986). Working memory. New York: Oxford University Press.

Baddeley, A. \& Hitch, G. (1974). Working memory. In Bower, G.A. The Psychology of Learning and Motivation. (pp. 47-89). New York: Academic Press.

Baddeley, A. \& Wilson, B. A. (2000). Prose recall and amnesia: implications for the structure of working memory. Neuropsychologia 40, 1737-1743.

Beck, I. L., Perfetti, C. A., \& McKeown, M. G. (1982). Effects of long-term vocabulary instruction on lexical access and reading comprehension. Journal of Educational Psychology, 74, 506-521.

Borella, E., Carretti, B., \& Pelegrina, S. (2010). The specific role of inhibition in reading comprehension in good and poor comprehenders. Journal of Learning Disabilities 43(6), 541-552

Cain, K. \& Oakhill, J. (2006). Profiles of children with specific reading comprehension difficulties. British Journal of Educational Psychology. 76(4), 683-696.

Cain, K. \& Oakhill, J. (2007). Children's comprehension problems in oral and written language: A Cognitive Perspective. The Guilford Press: London.

Cain, K., Oakhill, J., \& Bryant, P. E. (2004). Children's reading comprehension ability: Concurrent prediction by working memory, verbal ability, and component skills. Journal of Educational Psychology, 96, 31-42.

Canet-Juric, L., Urquijo, S., Richard's, M.M., \& Burin, D. (2009). Predictores cognitivos de niveles de comprensión lectora mediante análisis discriminante. International Journal of Psychological Research, 2(2), 99-111.

Carretti, B., Borella, E., Cornoldi, C., \& De Beni, R. (2009). Role of working memory in explaining the performance of individuals with specific reading comprehension difficulties: A meta-analysis. Learning and Individual Differences. 19(2), 245-251.

Cartoceti, R., Sampedro, B., Abusamra, V., \& Ferreres, A. (2009). Evaluación de la iniciación y la supresión de respuesta verbal en niños. Versión infantil en español del Test de Hayling. Fonoaudiológica, 55(2), 9-24.

Chiappe,P., Hasher, L., \& Siegel, L. (2000). Working memory, inhibitory control and reading disability. Memory \& Cognition 28(1), 8-17.

Cornoldi, C., De Beni, R., \& Pazzaglia, F. (1996). Profiles of reading comprehension difficulties: An analysis of single cases. In: Cornoldi, C.; Oakhill, J. (Eds), Reading comprehension difficulties: Processes and intervention (pp. 113136). Mahwah, NJ: Erlbaum.

Cutting, L., Eason, S., Young, K., \& Alberstadt, A. (2009). Reading comprehension: cognition and neuroimaging. In: Pugh, K.; McCardle, P., (Ed.). How children learn to read: current issues and new directions in the integration of cognition, neurobiology and genetics of reading and dyslexia research and practice (pp. 329) Philadelphia, PA: Earlbaum.

Daneman, M. \& Carpenter P. (1980). Individual differences in working memory and reading. Journal of Verbal Learning and Verbal Behavior, 19, 450-466.

Daneman, M. \& Merikle, P. (1996). Working memory and language comprehension: A meta-analysis. Psychonomic Bulletin \& Review, 3(4), 422-433.

De Beni, R., Borella, E., \& Carretti, B. (2007). Reading comprehension in aging: The role of working memory and metacomprehension. Aging, Neuropsychology, and Cognition. 14(2), 189-212.

De Beni, R., Pazzaglia, F., Gyselinck, V., \& Meneghetti, C. (2005).Visuospatial working memory and mental representation of spatial descriptions. European Journal of Cognitive Psychology. 17(1), 77-95.

Gernsbacher, M. A. (1990). Language comprehension as structure building. Hillsdale, NJ: Erlbaum.

Gutiérrez, F., García Madruga, J., Elosúa, R., Luque, J., \& Garate, M. (2002). Memoria operativa y comprensión lectora: algunas cuestiones básicas. Acción Psicológica, 1, 45-68.

Hasher, L., Lustig, C., \& Zacks, R. (2007). Inhibitory mechanisms and control of attention. In: Conway, A., Jarrold, C., Kane, M., Miyake, A \& Towse, J. (Eds.). Variation in Working Memory. (pp 227-249). New York: Oxford University Press. 
Hasher, L. \& Zacks, R. (1988). Working memory, comprehension, and aging: A review and a new view. In G. H. Bower (Ed.). The Psychology of Learning and Motivation, Vol. 22 (pp. 193-225). New York: Academic Press.

Hasher, L., Tonev, S., Lustig, C., \& Zacks, R. T. (2001). Inhibitory control, environmental support, and self-initiated processing in aging. In M. NavehBenjamin, M. Moscovitch, \& R. L. Roediger, III. (Eds.). Perspectives on Human Memory and Cognitive Aging: Essays in Honour of Fergus Craik (pp. 286-297). East Sussex, UK: Psychology Press.

Injoque-Ricle, I., Calero, A. D., Alloway, T. P., \& Burín, D. I. (2011). Assesing working memory in Spanish-speaking children: Automated Working Memory Assessment battery adaptation. Learning and Individual Differences, 21, 7884.

Kintsch, W. (1988). The role of knowledge in discourse processing: A constructionintegration model. Psychological Review, 95, 163-182.

Kintsch,W. (1998). Comprehension: A paradigm for cognition. New York: Cambridge University Press.

Matute, E., Rosselli, M., Ardila, A., \& Ostrosky-Solís, F. (2007). Evaluación Neuropsicológica Infantil (ENI). Manual de Aplicación. México: El Manual Moderno.

Miyake, A. \& Shah, P. (1999). Toward unified theories of working memory: Emerging general consensos, unresolved theoretical issues and future directions. En Miyake, A. y Shah (Eds.). Models of working memory: Mechanisms of active maintance and executive control (pp. 442-481). Cambridge: Cambridge University Press.

Morales-Vallejo, P. (2009). El tamaño del efecto (effect size): análisis complementarios al contraste de medias. Recuperado el 2 de mayo de 2012, del sitio Web http://www.upcomillas.es/personal/peter/

Oakhill, J. \& Yuill, N. (1996). Higher order factors in comprehension disability: Processes and remediation. In Cornoldi, J. \& Oakhill, J. (Eds.). Reading Comprehension Difficulties. Processes and Intervention (pp. 69-92). Mahwah, New Jersey: Earlbaum.

Perfetti, C. A. (1985). Reading ability. New York: Oxford University Press.

Pressley, M. (2000). What should comprehension instruction be the instruction of? In Kamil, M., Mosenthal, P., Pearson, P. \& Barr, R. (Eds.). Handbook of reading research: Volume III (pp. 545-561). Mahwah, NJ: Erlbaum.

Stelzer F., Cervigni, M., \& Martino, P. (2010). Bases neurales del desarrollo de las funciones ejecutivas durante la infancia y adolescencia. Una revisión. Revista Chilena de Neuropsicología. 5(3), 176-184.

Swanson, H. (1999). Reading comprehension and working memory in learningdisabled readers: Is the phonological loop more important than the executive system. Journal of Experimental Child Psychology, 72(1), 1-31.

Swanson, H. \& O'Connor, R. (2009). The role of working memory and fluency practice on the reading comprehension of students who are dysfluent readers. Journal of Learning Disabilities. 42(6), 548-575.

Watkins, M. W. (2003). Effect size calculator (Computer software). State College, PA Ed-Psych Associates.

Weshler, D. (1991). Test de Inteligencia para Niños WISC-III. Manual. Buenos Aires: Paidós.

Zacks, R. \& Hasher, L. (1994). Directed ignoring: Inhibitory regulation of working memory. In D. Dagenbach \& T. H. Carr (Eds.). Inhibitory mechanisms in attention, memory, and language (pp. 241-264). New York, NY: Academic Press. 